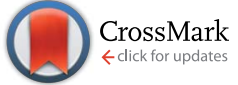

Cite this: RSC Adv., 2016, 6, 74100

Received 13th June 2016 Accepted 25th July 2016

DOI: 10.1039/c6ra15365h

www.rsc.org/advances

\title{
Friend or foe? The role of solvents in non-triplet, intraligand charge transfer sensitization of lanthanide(III) luminescence $\uparrow$
}

\author{
Wai-Sum Lo, Wing-Tak Wong* and Ga-Lai Law* \\ Sensitization of lanthanide luminescence in the visible and near infra-red (NIR) region was achieved via \\ a non-triplet energy transfer pathway by long-wavelength excitation of an intraligand charge transfer \\ (ILCT) band of a diethylamino-based ligand system. A comprehensive photophysical and solvatochromic \\ study is conducted to evaluate the luminescence properties of our Eu(III), Sm(III) and Yb(III) complexes. \\ The effects of solvent coordination and solvent quenching on lanthanide luminescence were studied in \\ detail and efforts were also devoted into examining the ambiguous energy transfer mechanism of $\mathrm{Yb}$ (III) \\ sensitization.
}

\section{Introduction}

Trivalent lanthanides $(\operatorname{Ln}(\mathrm{III}))$ are a "stubborn" but fascinating group of luminescent centers. Stubborn as in their inflexibility to absorb light, with very low extinction coefficients $(\varepsilon \approx 1-10 \mathrm{~L}$ $\left.\mathrm{mol}^{-1} \mathrm{~cm}^{-1}\right){ }^{1}$ which arises due to the Laporte-forbidden $\mathrm{f}-\mathrm{f}$ transitions. Therefore direct excitation is neither practical nor feasible in extensive applications as it often requires high-power lasers. Nevertheless, the forbidden nature imparts fascinating and unique luminescence characteristics to the $\operatorname{Ln}(\mathrm{III})$, such as long luminescence lifetimes ( $\mu \mathrm{s}$ to $\mathrm{ms}$ ), fingerprint emission profiles, fine emission bands and large pseudo-Stokes shifts. These properties have allowed lanthanides to reap benefits that organic chromophores and luminescent transition metal complexes lack and hence be applied in applications such as in displays and as optical imaging agents. ${ }^{1}$

The antenna effect is a general term describing the use of a chromophore to harvest light energy and transfer it to the $\mathrm{Ln}(\mathrm{III})$, followed by subsequent radiative decay to yield $\mathrm{f}-\mathrm{f}$ transitions and has become a common strategy for sensitizing lanthanide(III) luminescence. There are several energy transfer pathways from the chromophore to the Ln(III) center, with the most common one involves an intersystem crossing process facilitated by heavy atom effect of $\operatorname{Ln}(\mathrm{III})$ - to populate the triplet excited state followed by energy transfer to the Ln(III) accepting state(s). ${ }^{1}$ The long-lived triplet state allows more time for the excited energy to be transferred to the $\operatorname{Ln}(\mathrm{III})$ but the energy difference between the photoexcitation and the accepting

Department of Applied Biology and Chemical Technology, The Hong Kong Polytechnic University, Hung Hom, Hong Kong, China. E-mail: w.t.wong@polyu.edu.hk; ga-lai. law@polyu.edu.hk

$\dagger$ Electronic supplementary information (ESI) available. See DOI: 10.1039/c6ra15365h state(s) would have to be large to accommodate for intersystem crossing. An energy pathway circumventing the triplet excited state would allow lower energy excitation and thus be more advantageous in various applications.

The energy transfer mechanism of the sensitization process is worthy of fundamental research interest, too, as the physical prerequisites for different non-mutually exclusive mechanisms - Förster ${ }^{2}$ and Dexter ${ }^{3}$ - is closely correlated to the structural design of the chromophore, such as spectral overlap in FRET, and Ln(III)-chromophore distances. The ability to understand and manipulate such relationship(s) would help establish suitable blueprints for designated applications. The role of luminescence quenchers such as high-energy $\mathrm{O}-\mathrm{H}, \mathrm{C}-\mathrm{H}$ oscillators with harmonics of resonant energies with the $\Delta E$ of $\operatorname{Ln}($ III) should also be taken into account when designing the complex. ${ }^{4}$

In this work, we nominated three Ln(III) for our investigation: $\mathrm{Eu}(\mathrm{III})$ is chosen for its bright luminescence in the visible region and its hypersensitivity transition for studying the surrounding environment; ${ }^{5} \mathrm{Sm}$ (III) for having an accepting state of similar energy to $\mathrm{Eu}(\mathrm{III})$ and its ability to emit in both the visible and NIR region; ${ }^{6}$ and $\mathrm{Yb}(\mathrm{III})$ for its low-energy NIR emission and intriguing energy transfer pathway. ${ }^{6 a, 7}$ The three $\operatorname{Ln}(\mathrm{III})$ was complexed with the same ligand system which consists of three 2-thenoyltrifluoroacetonate (TTA) moieties - a known, good antenna that sensitizes all three Ln(III) through the tripletmediated energy transfer pathway ${ }^{8}$ - and a neutral tridentate scaffold with a diethylamino moiety imparting intraligand charge transfer (ILCT) character, which is able to sensitize $\mathrm{Eu}(\mathrm{III})^{9}$ and $\mathrm{Sm}(\mathrm{III})^{10}$ luminescence via a non-triplet mediated pathway. This current work extends the scope of our previous studies, expanding the work with different Ln(III) emitters as well as with various solvents of different polarity in an attempt to investigate the various processes mentioned above. 


\section{Results and discussion}

The complexes were synthesized as previously reported (Scheme 1), ${ }^{10}$ where ligand 1 and the $\operatorname{Ln}(\mathrm{tta})_{3} \cdot x \mathrm{H}_{2} \mathrm{O}$ (either commercially available or as-synthesized), were mixed in a $1: 1$ stoichiometry in methanol at $50{ }^{\circ} \mathrm{C}$ for 16 hours. The crude was dissolved in minimum amount of diethyl ether and precipitated by $n$-hexanes for three cycles to obtain the product as yellowish solids. Different Ln(III) complexes exhibit identical solubilities, notably insoluble only in $n$-hexanes, petroleum ether and water.

\section{UV-vis absorption and excitation spectra}

The absorption spectra of the complexes in solution displayed three absorption bands in the ultraviolet region. The highest energy band at ca. $260 \mathrm{~nm}$ is assigned as the $\pi-\pi^{*}$ transition of the dipyrazolyltriazine scaffold and the band with a maximum at ca. $340 \mathrm{~nm}$ is assigned as the $\pi-\pi^{*}$ transition of the TTA moiety. The ILCT absorption band, which shifts with solvent polarity, could be observed with a maximum ranging from $c a .350 \mathrm{~nm}$ to $420 \mathrm{~nm}$. The polar ground state of the molecule (donor-acceptor nature of the diethylaniline and dipyrazolyltriazine ends) imparts negative solvatochromism properties to the compound. Polar solvents stabilizes the polar ground state and hence the $\Delta E$ becomes larger, resulting in a hypsochromic shift. ${ }^{11}$ The excitation of the ILCT band does not necessarily sensitize lanthanide(III) luminescence, though, as the fluorescence may not be at a suitable energy level for energy transfer to take place. We have compared the UV-vis absorption spectra and the excitation spectra of the complexes to illustrate this finding.

As seen in the excitation spectra (Fig. 1 and ESI Fig. S1-S12, S25-S37 and S50-S61†), all the complexes have an excitation band with a maximum at $c a .340 \mathrm{~nm}$, which has a nearly identical band shape and maximum with the absorption band of the TTA moiety, indicating antenna effect via the TTA. In nonpolar solvents (benzene, toluene, carbon tetrachloride), an additional excitation band which is superimposable with the ILCT absorption band could be found, confirming sensitization via excitation of the ILCT band as well, which is a non-triplet energy transfer pathway. ${ }^{9}$ The difference between the intensities of the absorption and excitation implies that the excitation of the ILCT ligand is more efficient in sensitizing lanthanide luminescence than the three moieties of TTA. Similar

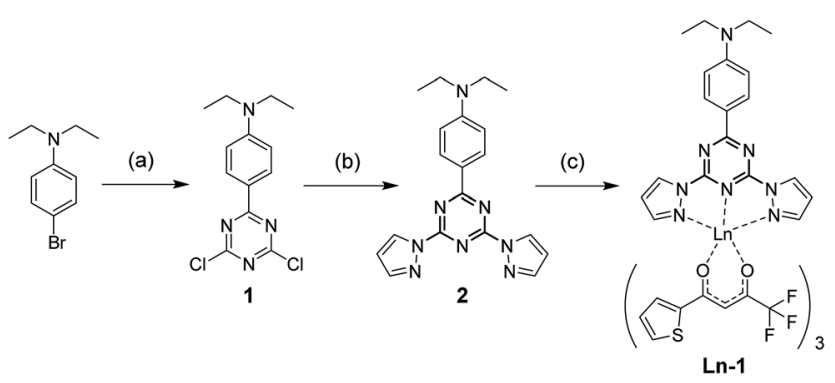

Scheme 1 Synthesis of Ln-1. (a) (i) Mg, I2, THF; (ii) cyanuric chloride, THF, reflux, $8 \mathrm{~h}$ (b) potassium pyrazolide, THF, reflux, $16 \mathrm{~h}$. (c) $\operatorname{Ln}(\mathrm{tta})_{3}$, $\mathrm{MeOH}, 50^{\circ} \mathrm{C}, 12$ hours.

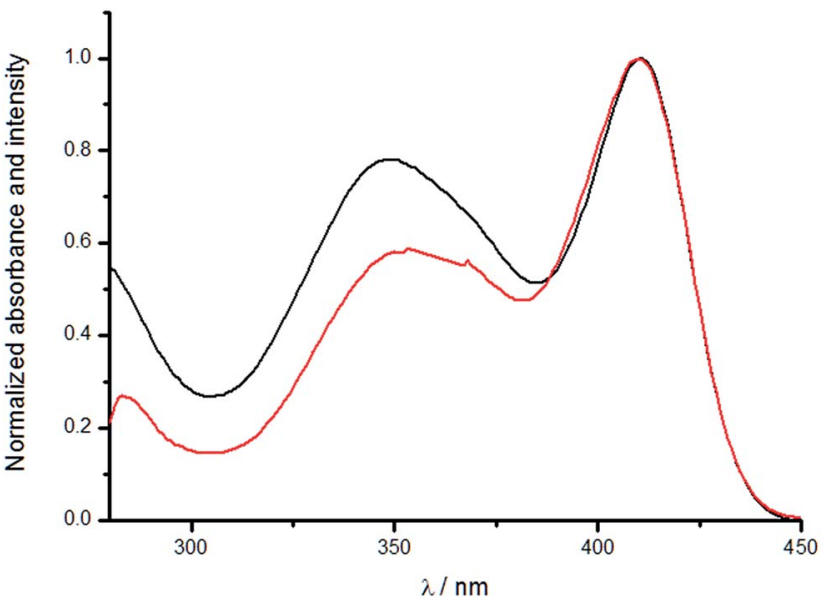

Fig. 1 Normalized absorption (black) and excitation (red) spectra of Eu-1 in benzene $\left(\lambda_{\mathrm{em}}=620 \mathrm{~nm}\right)$.

observations could be obtained from the spectra of chloroform, dichloromethane and 1,2-dichloroethane as well, albeit with a consistent red-shift of the excitation peak maxima compared to the absorption peak maxima.

The absorption spectra in polar solvents, on the other hand, mostly presented one broad band which we assign as a merged band of the TTA moiety and the blue-shifted ILCT band (vide supra). However, the excitation band only revealed a single band which is from the TTA as its maximum is at $c a .340 \mathrm{~nm}$. The results indicate that the ILCT band could not effectively sensitize the $\operatorname{Ln}(\mathrm{III})$ in polar environments.

The excitation spectra of the three complexes are very similar, as expected of the ligand comprising of the same chemical components. For Sm(III) complexes, the excitation spectra of the visible $\left({ }^{4} \mathrm{G}_{5 / 2} \rightarrow{ }^{6} \mathrm{H}_{9 / 2}\right)$ and NIR $\left({ }^{4} \mathrm{G}_{5 / 2} \rightarrow{ }^{4} \mathrm{~F}_{5 / 2}\right)$ transitions are also nearly identical in spectral-terms of both band shape and intensities. The results are as expected as both transitions decay from the same excited ${ }^{4} \mathrm{G}_{5 / 2}$ level, as typically excitation spectra only take in account of where the excited energy is from and not where it ends.

In the case of acetonitrile (Fig. 2), the ILCT absorption band is rather distinct $\left(\lambda_{\max } c a .420 \mathrm{~nm}\right)$, despite it being a polar

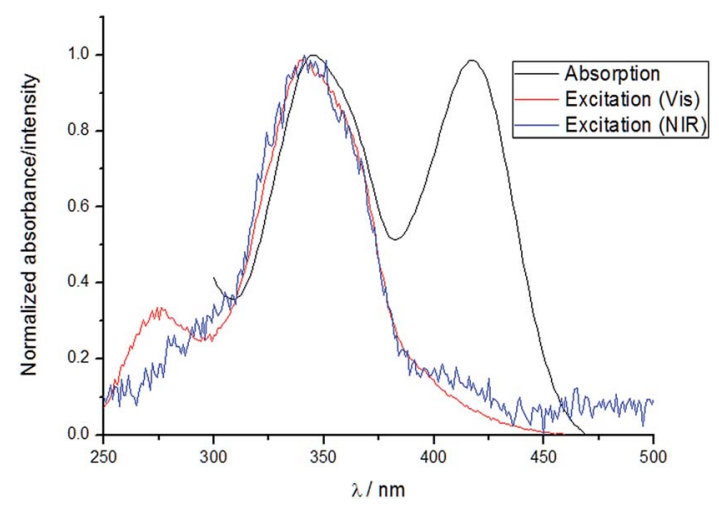

Fig. 2 Normalized absorption and excitation spectra of Sm-1 in acetonitrile ( $\lambda_{\mathrm{em}}=645 \mathrm{~nm}$ (vis), $950 \mathrm{~nm}$ (NIR)). 
solvent $(\mu=3.92)$. It is due to the highly polarizing nature of acetonitrile $(\varepsilon=37.5)$ which increases the rate of charge transfer from the diethylamino end to the dipyrazolyltriazine end such that stabilization by solvent dipole - which does not reach an electrostatic equilibrium with the molecule instantaneously - is rendered insignificant. This also explains the shoulders in the absorption spectra of the complexes in acetone $(\varepsilon=20.7)$ and $\operatorname{DMSO}(\varepsilon=46.7)$.

\section{Emission spectra}

The complexes were excited at three different wavelengths (330, $350,390 \mathrm{~nm})$. Excitation at 330 and $350 \mathrm{~nm}$ should give consistent results as the involvement of ILCT sensitization should be minimum, whereas the TTA moieties would be irrelevant with excitation of the ILCT band at $390 \mathrm{~nm}$. The trend provides comprehensive data for comparison of different sensitization moieties. By studying the emission spectra of our $\mathrm{Eu}(\mathrm{III})$ complex, the hypersensitive ${ }^{5} \mathrm{D}_{0} \rightarrow{ }^{7} \mathrm{~F}_{2}$ transition were found to be different in different solvents, hinting at different coordination environments around the emitting Eu(III) ion. ${ }^{\mathbf{1 2}}$ In non-polar or less polar solvents, the splitting pattern of the hypersensitive transition peaks are quite similar, regardless of the energy transfer pathway undertaken prior to radiative decay (Fig. 3). As the solvent becomes more polar, the donor atom of the solvent molecules with increased nucleophilicity coordinate to the $\mathrm{Ln}$ (III) center and displace the neutral tridentate triazinebased scaffold. This could be reflected by the inconsistency in the splitting patterns in polar solvents. As the solvent molecules coordinate onto the Eu(III) center, the coordination environment varied to different extents depending on the number of molecules coordinated as well as the steric effect imposed on the overall structure.

In polar solvents, while the splitting patterns are consistent between 330 and $350 \mathrm{~nm}$ excitation, they differ when excited at $390 \mathrm{~nm}$. We believe that it is due to the remaining unperturbed complex molecules that retained the tridentate ligand in their structures to give considerably weaker luminescence via ILCT excitation.

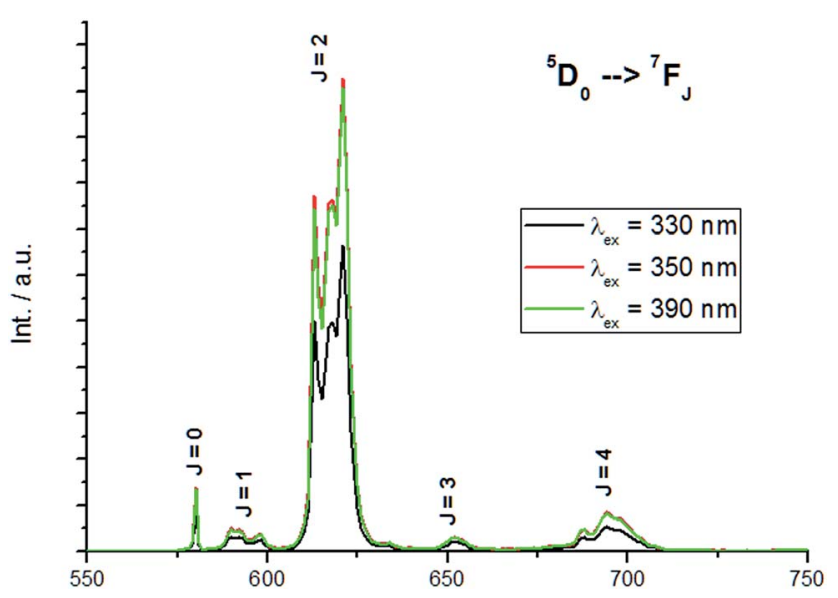

Fig. 3 Room temperature emission spectra of Eu-1 in benzene at 0.1 absorbance (455 $\mathrm{nm}$ long pass filter).
Of the ${ }^{5} \mathrm{D}_{0} \rightarrow{ }^{7} \mathrm{~F}_{1}$ and ${ }^{5} \mathrm{D}_{0} \rightarrow{ }^{7} \mathrm{~F}_{2}$ transitions of $\mathrm{Eu}(\mathrm{III})$, the former transition is primarily magnetic dipole in nature and is largely independent of the ligand environment, acting as an internal standard for comparison with the latter hypersensitive transition. The asymmetry ratio, defined as the ratio between the integrated intensities of the two transitions provides an additional parameter to understand the effect of solvent coordination $^{\mathbf{1 2}}$ (the asymmetry ratio is intrinsically high due the unrestricted coordination manner of the three TTA molecules). A big difference can be observed between the non-polar and polar solvents, especially methanol and DMSO with high coordination strength. However, it must also be mentioned that similar asymmetry ratios have no implications of similar coordination environments, i.e. does not rule out solvent coordination in this case. The asymmetry ratio when excited at $390 \mathrm{~nm}$ also reinforces our above postulate by evidencing two emitting species of different extents of asymmetry.

Similarly, the emission intensities of $\mathbf{S m - 1}$ in non-polar solvents via excitation at the ILCT band are comparable with TTA excitation (Fig. 4). The same trend in intensities with Eu-1 is observed as well due to the similar energy levels of their excited states. Yet the intrinsically weak quantum efficiency of Sm(III), ${ }^{6,10}$ solvent quenching and masking by strong ligand fluorescence are reasons behind the absence of any observable Sm(III) emission profile in certain polar solvents. While the hypersensitive transition ${ }^{4} \mathrm{G}_{5 / 2} \rightarrow{ }^{6} \mathrm{H}_{9 / 2}$ is not as sensitive as that of Eu(III), the asymmetry ratio between it and the ${ }^{4} \mathrm{G}_{5 / 2} \rightarrow{ }^{6} \mathrm{H}_{5 / 2}$ transition - predominantly magnetic dipole in nature - gives similar information as that of the Eu(III) counterpart and the results are summarized in Table 1 ; the same trend and explanation apply.

The results are very similar for $\mathbf{Y b - 1}$, too. The unique emission profile from $900-1050 \mathrm{~nm}$ with a peak maximum at ca. 980 nm (Fig. 5), observed via both TTA and ILCT excitation, is attributed to the ${ }^{2} \mathrm{~F}_{5 / 2} \rightarrow{ }^{2} \mathrm{~F}_{7 / 2}$ transition, which is split into several components as a result of the ligand-field effect ${ }^{7}$ on both

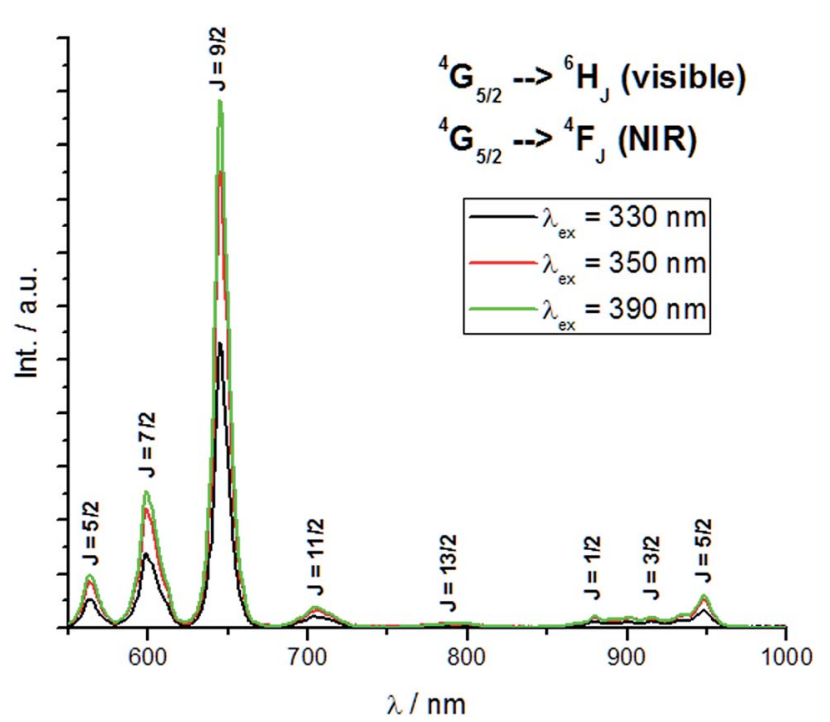

Fig. 4 Room temperature emission spectra of Sm-1 in toluene at 0.3 absorbance (530 $\mathrm{nm}$ long pass filter). 
Table 1 Ratios of integrated intensities of selected transitions, visible luminescence quantum yields and lifetimes (estimated error of $\pm 15 \%$ and $10 \%$ respectively) of Eu-1 and Sm-1 in various solvents

\begin{tabular}{|c|c|c|c|c|c|c|c|c|c|}
\hline Solvents & $\mu(\mathrm{D})$ & $\begin{array}{l}\mathbf{E u}-\mathbf{1} R^{a} \\
\left(\lambda_{\mathrm{ex}}=350 \mathrm{~nm}\right)\end{array}$ & $\begin{array}{l}\text { Eu-1 } R^{a} \\
\left(\lambda_{\mathrm{ex}}=390 \mathrm{~nm}\right)\end{array}$ & $\begin{array}{l}\text { Sm-1 } R^{b} \\
\left(\lambda_{\mathrm{ex}}=350 \mathrm{~nm}\right)\end{array}$ & $\begin{array}{l}\text { Sm-1 } R^{b} \\
\left(\lambda_{\mathrm{ex}}=390 \mathrm{~nm}\right)\end{array}$ & Eu-1 $\tau^{c}$ & Sm-1 $\tau^{d}$ & $\begin{array}{l}\text { Eu-1 } \Phi^{e} \\
(\%)\end{array}$ & $\begin{array}{l}\operatorname{Sm-1} \Phi^{e} \\
(\%)\end{array}$ \\
\hline $\mathrm{CCl}_{4}$ & 0 & 18.4 & 18.5 & 10.8 & 12.8 & 0.494 & 0.159 & $d$ & \\
\hline Benzene-d6 & 0 & & & & & - & 0.124 & - & 5.03 \\
\hline Toluene & 0.37 & 19.0 & 19.2 & 10.7 & 10.5 & 0.425 & 0.0935 & 30.6 & 3.13 \\
\hline Toluene-d8 & 0.37 & & & & & - & 0.115 & - & 6.27 \\
\hline $\mathrm{CH}_{2} \mathrm{Cl}_{2}$ & 1.6 & 14.3 & 12.4 & 5.4 & 3.8 & $0.013 ; 0.196$ & $0.022 ; 0.121$ & $f$ & \\
\hline $\mathrm{CH}_{3} \mathrm{OH}$ & 1.7 & 12.0 & - & 3.0 & - & 0.2260 .547 & 0.016 & 2.9 & 0.135 \\
\hline $\mathrm{CD}_{3} \mathrm{OD}$ & 1.7 & & & & & $0.315 ; 1.109$ & 0.131 & - & \\
\hline THF & 1.75 & 16.6 & - & 5.3 & - & 0.383 & 0.027 & 10.7 & 1.21 \\
\hline EA & 1.78 & 16.0 & 6.5 & 3.7 & - & $0.187 ; 0.397$ & 0.059 & 7.1 & 1.36 \\
\hline DCA & 1.8 & 17.1 & 11.6 & 4.6 & - & $0.114 ; 0.287$ & $0.017 ; 0.092$ & $f$ & \\
\hline
\end{tabular}

${ }^{a} I\left({ }^{5} \mathrm{D}_{0} \rightarrow{ }^{7} \mathrm{~F}_{2}\right) / I\left({ }^{5} \mathrm{D}_{0} \rightarrow{ }^{7} \mathrm{~F}_{1}\right) \cdot{ }^{b} I\left({ }^{4} \mathrm{G}_{5 / 2} \rightarrow{ }^{6} \mathrm{H}_{9 / 2}\right) / I\left({ }^{4} \mathrm{G}_{5 / 2} \rightarrow{ }^{6} \mathrm{H}_{5 / 2}\right)$. Blank entries are due to interference to transition intensities from ligand residual fluorescence (see ESI). ${ }^{c}$ Emission lifetime of the ${ }^{5} \mathrm{D}_{0} \rightarrow{ }^{7} \mathrm{~F}_{2}$ transition. ${ }^{d}$ Emission lifetime of the ${ }^{4} \mathrm{G}_{5 / 2} \rightarrow{ }^{6} \mathrm{H}_{9 / 2}$ transition. ${ }^{e}$ Relative to quinine sulfate in $0.1 \mathrm{M} \mathrm{H}_{2} \mathrm{SO}_{4},\left(\lambda_{\mathrm{ex}}=350 \mathrm{~nm} ; \Phi=0.577\right) .{ }^{f}$ Instability upon dilution, inappropriate for measurement. Some of the $\mathrm{Sm}^{\mathrm{III}}$ data is taken from ref. 10 .

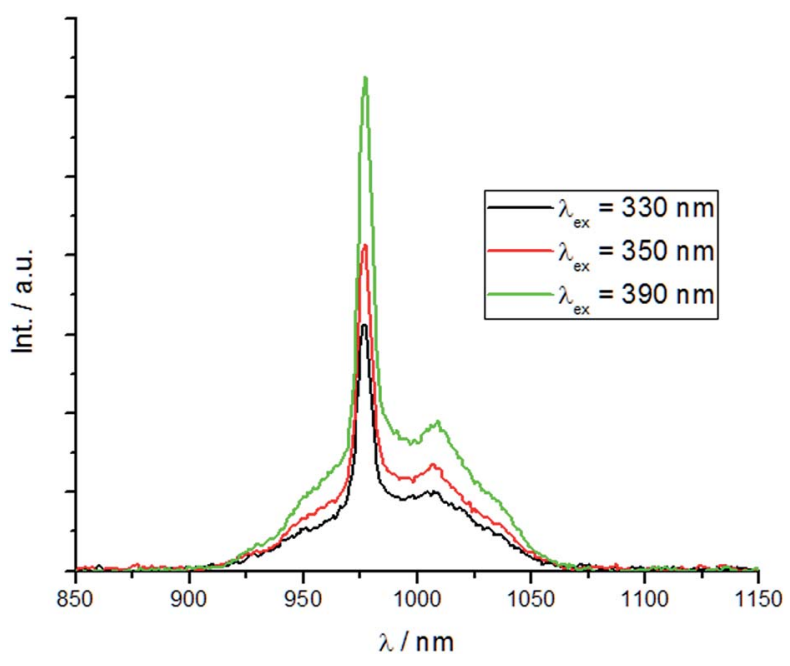

Fig. 5 Room temperature emission spectra of $\mathrm{Yb}-1$ in toluene at 0.3 absorbance (830 $\mathrm{nm}$ long pass filter) showing the ${ }^{2} \mathrm{~F}_{5 / 2} \rightarrow{ }^{2} \mathrm{~F}_{7 / 2}$ transition.

the emitting and ground states. Yb(III) luminescence via excitation of ILCT band in non-polar solvents were of comparable intensities to TTA excitation yet by comparing the absorption and excitation spectra (ESI, Fig. S50-S61†), the less smooth excitation curves - even in non-polar solvents - indicated very weak sensitization by antenna effect.

\section{Energy transfer pathway}

One of the main objectives of this work is to discover more about the ILCT band and the role it plays and could play in sensitizing $\operatorname{Ln}(\mathrm{III})$ luminescence. TTA is a well-known $\beta$-diketone capable of sensitizing $\mathrm{Eu}(\mathrm{III}), \mathrm{Sm}$ (III) and $\mathrm{Yb}$ (III) luminescence and the purpose of incorporating it in our complexes is to serve as an internal standard of the antenna effect. Low temperature $(77 \mathrm{~K})$ measurements of $\mathbf{G d - 1}$ and $\mathrm{Gd}(\mathrm{tta})_{3}$ were done in 2methyltetrahydrofuran in an attempt to measure the triplet states of the complex. Gd(III) was chosen as it is able to simulate the coordination environment of the other complexes - due to the similar ionic radii across the lanthanides - while eliminating the possibility of energy transfer to lanthanide(III) upon ligand excitation as its only excited state is located beyond $30000 \mathrm{~cm}^{-1}$. Excitation at $350 \mathrm{~nm}$ yielded a lower energy band (ESI, Fig. S74†) in Gd-1 with an emission lifetime of $3.6 \mathrm{~ms}$. Comparison with $\mathrm{Gd}(\mathrm{tta})_{3}$ further confirms the assignment as the TTA triplet. An additional higher energy emission band with a maximum at $c a .400 \mathrm{~nm}$ was observed in which a biexponential lifetime was obtained (3.6 and $10 \mu \mathrm{s}$ ). When Gd-1 was excited at $390 \mathrm{~nm}$ (Fig. 6), the phosphorescence from TTA was absent and the same higher energy emission band $(\tau=9.3$ and $1.8 \mu \mathrm{s}$ ), assigned as the fluorescence of ILCT transition, was observed with a shoulder at $c a .422 \mathrm{~nm}(\tau=10$ and $1.6 \mu \mathrm{s})$.

As the antenna effect via the TTA moiety (triplet-mediated pathway) is well documented in literature, we would like to focus on the sensitization by excitation the ILCT band. A simplified Jablonski diagram is presented in Fig. 7 with the triplet energy level of TTA, the ILCT band and the accepting states of $\mathrm{Eu}(\mathrm{III}), \mathrm{Sm}$ (III) and $\mathrm{Yb}$ (III). The non-triplet ILCT excited state energy was determined to be $c a .23900 \mathrm{~cm}^{-1}$ by room temperature emission spectrum of Gd-1. The ${ }^{5} \mathrm{D}_{2}$ and ${ }^{5} \mathrm{D}_{1}$ accepting states of $\mathrm{Eu}(\mathrm{III})$ are at ca. 21500 and $19200 \mathrm{~cm}^{-1}$ respectively, and are seemingly more preferable destinations for energy transfer from the ILCT level due to a smaller energy 


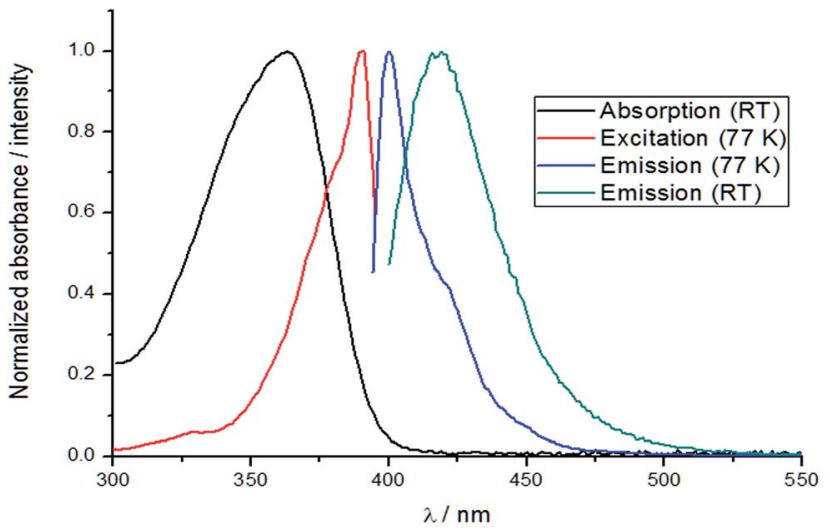

Fig. 6 Normalized room temperature absorption and emission spectra $\left(\lambda_{\mathrm{ex}}=390 \mathrm{~nm}\right)$ and low temperature excitation $\left(\lambda_{\mathrm{em}}=400 \mathrm{~nm}\right)$ and emission $\left(\lambda_{\text {ex }}=390 \mathrm{~nm}\right.$ ) spectra of Gd-1 in 2-MeTHF.

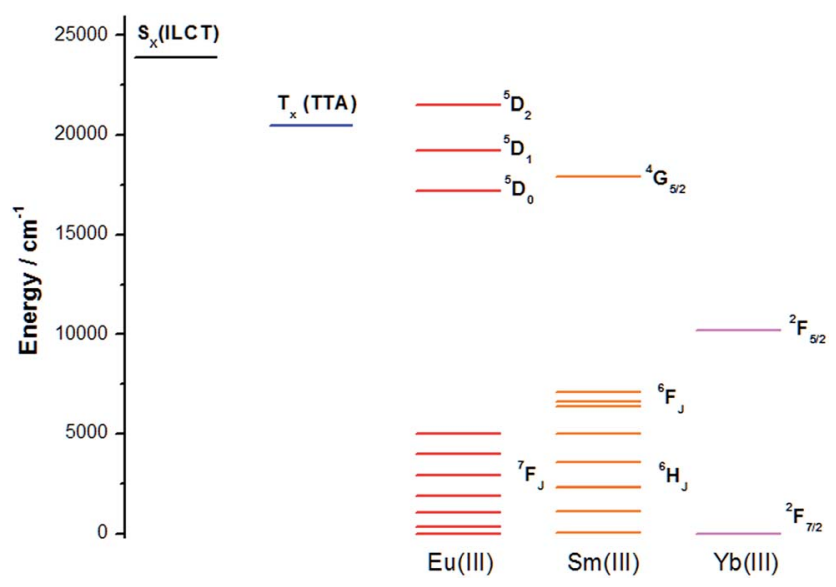

Fig. 7 Simplified Jablonski diagram depicting energy levels of chromophores and of $\mathrm{Ln}^{\prime \prime \prime}$.

gap, ${ }^{13}$ compared to ${ }^{5} \mathrm{D}_{0}$ (ca. $17200 \mathrm{~cm}^{-1}$ ), although energy transfer to the latter should not be ruled out as the ${ }^{4} \mathrm{G}_{5 / 2}$ accepting state (ca. $17900 \mathrm{~cm}^{-1}$ ) of $\mathrm{Sm}$ (III) is also a recipient of the excited energy in $\mathbf{S m - 1}$. The energy of the excited state, however, is expected to change in different polarities. The less polar excited state will be stabilized to a larger extent than the polar ground state, thus reducing the gap with the accepting states, facilitating energy transfer. Considering other factors such as displacement of the ILCT ligand as well as quenching by oscillators of solvent molecules, various non-radiative processes dominate the energy transfer process hence resulting in the observed trend.

On the other hand, the spectral overlap between the ILCT band and the absorption of $\mathrm{Yb}(\mathrm{III})$ is minimal, especially without a lower energy triplet state. The energy transfer mechanism in the sensitization of Yb-1 may therefore be majorly a long range electron transfer process analogous to Horrocks Jr's work. ${ }^{7 a}$ Excitation of Yb-1 is believed to have caused an intramolecular electron transfer from the diethylamino moiety to the dipyrazolyltriazine and form a temporary charge-separated state: positive and negative charge at the donor and acceptor respectively. The extended Rehm-Weller equation ${ }^{14}$ could indicate whether this electron-transfer process is thermodynamically favorable:

$$
\Delta G_{\mathrm{ET}}=\left(E_{\mathrm{ox}}-E_{\mathrm{red}}\right)-E_{\mathrm{s}}-e_{\mathrm{o}}^{2} / \varepsilon_{\mathrm{a}}
$$

where $\Delta G_{\mathrm{ET}}$ is the change in free energy upon electron transfer, $E_{\mathrm{ox}}$ and $E_{\text {red }}$ are the oxidation potential of the donor (ligand of complex) and reduction potential of the acceptor $\left(\mathrm{Yb}^{3+}\right)$ respectively, as determined by cyclic voltammetry experiments with Yb-1 (Fig. S75 $\dagger$ ). $E_{\mathrm{s}}$ is the singlet state energy (zero-zero transition) of the ligand (determined by averaging the longest excitation maximum and the shortest emission maximum at 77 $\mathrm{K})$ and $e_{\mathrm{o}}{ }^{2} / \varepsilon_{\mathrm{a}}$ is the coulombic attraction experienced by the radical ion pair (taken as $0.15 \mathrm{eV}$ ). ${ }^{7 b, 7 e, 12}$ For $\mathbf{Y b - 1}$, the $\Delta G_{\mathrm{ET}}$ is calculated as: $(0.77+1.55)-3.1-0.15=-0.93 \mathrm{eV}$ and the electron transfer process is feasible. Along with our results of not finding any triplet states from the ligand in the low temperature measurements, we propose that a certain degree of sensitization of $\mathrm{Yb}(\mathrm{III})$ luminescence may result from a fast, long range electron transfer from the ligand to the $\mathrm{Yb}$ (III) center.

\section{Luminescence lifetimes and quantum yields}

Quantum yields in chlorinated solvents were not measured as instability issues were observed. ${ }^{10,15}$ In chloroform, dichloromethane, dichloroethane, chlorobenzene, 1,2-dichlorobenzene and carbon tetrachloride, the complexes all suffer from an abnormal deterioration of their absorption spectra - either after prolonged idle time or dilution (Fig. S76-S79†). These solvents possess relative low dipole moments, and complexes at initial dissolution displayed the ILCT absorption bands. However, as time passes or, more severely, upon dilution (e.g. in relative quantum yield measurements), the ILCT band deteriorates the ILCT absorbance plummets and $\lambda_{\max }$ blue-shifts simultaneously - much quicker than the TTA part. Water was dismissed as a possible cause as the band deterioration problem was not observed in water-miscible solvents. The presence of hydrogen chloride, the main decomposition product in chlorinated solvents, was also suspected as the interference. The solvents were therefore pre-treated with different bases: sodium bicarbonate, potassium carbonate and calcium hydride. However, the problem persisted after the solvents were treated for 2 hours or overnight with the bases, therefore we decided to take the results in these solvents as reference only.

Dipole moment was used as the parameter to relate solvents with their polarity and nucleophilicity. Under excitation of the TTA moiety, both Eu-1 and Sm-1 exhibit a general trend in which the quantum yields decreases with an increase of the dipole moment of the solvent molecules (Chart 1). The aromaticity of benzene and toluene provides rigidity to the ligand skeleton and minimizes vibronic deactivation, protecting the Ln(III) center well.

The solvent molecules interacting with the $\mathrm{Ln}$ (III) in the first coordination sphere, though, play an equally significant role. They could readily quench the luminescence via high energy vibrational overtones of $\mathrm{O}-\mathrm{H}$ and $\mathrm{C}-\mathrm{H}$ oscillators. The quenching effect is commonly explained by the energy gap law 


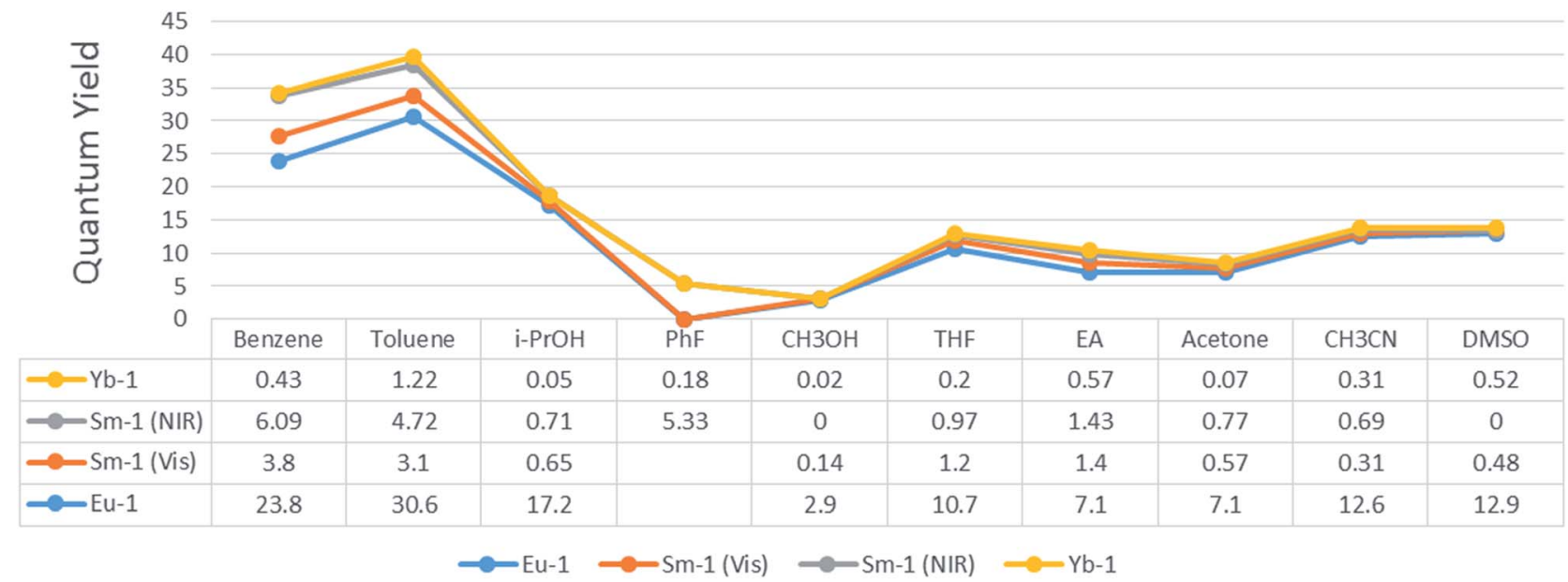

Chart 1 Visible luminescence quantum yield of Eu-1 and Sm-1 (vis) relative to quinine sulfate in $0.1 \mathrm{M} \mathrm{H}_{2} \mathrm{SO}_{4}\left(\lambda_{\text {ex }}=350 \mathrm{~nm}, \Phi=0.577\right)$ and NIR luminescence quantum yields of Sm-1 and $\mathrm{Yb}-1$ in various solvents relative to $\mathrm{Yb}(\mathrm{tta})_{3}\left(\mathrm{H}_{2} \mathrm{O}\right)_{2}$ in toluene $\left(\lambda_{\mathrm{ex}}=340 \mathrm{~nm}, \Phi=0.35 \%\right)(\mathrm{estimated}$ error is $\pm 15 \%$ ). Values of visible luminescence quantum yield of Sm-1 are taken from ref. 10 .

(EGL),$^{16}$ which denotes the nonradiative quenching by vibrational overtones of high energy oscillators matching well with the energy gap between the excited state and the next lower energy state of the $\operatorname{Ln}(\mathrm{III})$. This multiphonon energy transfer process is analogous to the Förster resonance energy transfer and thus exhibits a distance-dependent relationship $\left(\alpha 1 / r^{6}\right)$. The EGL is prevalently applied to explain the quenching lanthanide luminescence since the electronic states of the lanthanides remain unaffected in different environments. ${ }^{1}$

To investigate such effect, lifetime measurements were done in selected deuterated solvents (Table 2). Some of the data in non-deuterated solvents for visible luminescence lifetimes of Sm-1 could be found in our previous publication. ${ }^{10}$ (It should also be noted that only the shorter lifetime the bi-exponential

Table 2 NIR luminescence quantum yields (estimated error of $\pm 15 \%$ ) of $\mathrm{Sm}-1$ and $\mathrm{Yb}-1$ in various solvents

\begin{tabular}{llll}
\hline Solvent & $\mu(\mathrm{D})$ & Sm-1 $\Phi^{a}(\%)$ & Yb-1 $\Phi^{b}(\%)$ \\
\hline Benzene & 0 & 6.09 & 0.43 \\
Benzene-d6 & 0 & 2.69 & 0.52 \\
Toluene & 0.37 & 4.72 & 1.22 \\
Toluene-d8 & 0.37 & 3.42 & 0.99 \\
i-PrOH & 1.56 & 0.71 & 0.05 \\
Fluorobenzene & 1.66 & 5.33 & 0.18 \\
$\mathrm{CH}_{3} \mathrm{OH}$ & 1.7 & $b$ & 0.02 \\
$\mathrm{CH}_{3} \mathrm{OD}$ & 1.7 & 0.12 & 0.34 \\
$\mathrm{CD}_{3} \mathrm{OD}$ & 1.7 & 1.37 & 1.05 \\
$\mathrm{THF}^{\mathrm{EA}}$ & 1.75 & 0.97 & 0.20 \\
$\mathrm{Acetone}$ & 1.78 & 1.43 & 0.57 \\
$\mathrm{CH}_{3} \mathrm{CN}$ & 2.88 & 0.77 & 0.07 \\
$\mathrm{CD}_{3} \mathrm{CN}$ & 3.92 & 0.69 & 0.31 \\
$\mathrm{DMSO}$ & 3.92 & $c$ & 1.74 \\
\end{tabular}

${ }^{a}$ Relative to $\mathrm{Yb}(\mathrm{tta})_{3}$ in toluene $\left(\lambda_{\mathrm{ex}}=340 \mathrm{~nm}, \Phi=0.35 \%\right)$. ${ }^{b}$ Luminescence intensity too weak to give a satisfactory curve for calculations upon dilution. ${ }^{c}$ Instability upon dilution, inappropriate for measurement. lifetimes were reported in ref. 10 as we did not look into the instability issues at that time.) Since the NIR luminescence of Sm-1 and Yb-1 are too weak for lifetime measurements, their quantum yields were measured instead (Chart 1), as quantum yield and luminescent lifetime are in a proportional relation$\operatorname{ship}\left(\Phi=\tau k_{\mathrm{r}}\right)$.

Methanol and acetonitrile molecules are expected to coordinate to the $\operatorname{Ln}(\mathrm{III})$ center and therefore in methanol- $\mathrm{d}_{4}$ and acetonitrile- $\mathrm{d}_{3}$ (for $\mathbf{Y b}-\mathbf{1}$ only, as instability issues similar to the chlorinated solvents were observed in repeated trials of Sm-1), the quantum yields are greatly enhanced from non-deuterated solvents. In methanol- $\mathrm{d}_{1}\left(\mathrm{CH}_{3} \mathrm{OD}\right)$, the quantum yields of both complexes increased as well but were still some way off from methanol- $\mathrm{d}_{4}$. These results proved that, for donor molecules in the first coordination sphere, the presence of $\mathrm{O}-\mathrm{H}$ oscillators quenches the luminescence but the extent of quenching by proximal C-H oscillators is more pronounced. The energy gaps between the emitting state and the next closest ground state of the Ln(III) are: $\Delta E\left({ }^{4} \mathrm{G}_{5 / 2} \rightarrow{ }^{6} \mathrm{~F}_{11 / 2}\right)$ of $\operatorname{Sm}(\mathrm{III}): \approx 7400 \mathrm{~cm}^{-1}$ and $\Delta E\left({ }^{2} \mathrm{~F}_{5 / 2} \rightarrow{ }^{2} \mathrm{~F}_{7 / 2}\right)$ of $\mathrm{Yb}(\mathrm{III}): \approx 10200 \mathrm{~cm}^{-1}$. The drastic increase $(>15 \times)$ in quantum yields of Yb-1 by deuteration of $\mathrm{O}-\mathrm{H}$ in methanol can be explained by eliminating the efficient multiphonon quenching by the second $\mathrm{O}-\mathrm{H}$ vibrational overtone $\left(\approx 10500 \mathrm{~cm}^{-1}\right)$, justifying the EGL. The relatively slight increase $\left(3 \times\right.$ in $\mathrm{CD}_{3} \mathrm{OD}$ and $6 \times$ in $\left.\mathrm{CD}_{3} \mathrm{CN}\right)$ by deuteration of $\mathrm{C}-\mathrm{H}$ is due to the less efficient matching of the third $\mathrm{C}-\mathrm{H}$ overtone $\left(\approx 11600 \mathrm{~cm}^{-1}\right)$ with $\Delta E$.

The quantum yield results of $\mathbf{S m - 1}$, however, could not fit in the EGL preposition. The energy gap of Sm(III) did not match any overtones of the oscillators. The second and third aliphatic $\mathrm{C}-\mathrm{H}$ overtones are at $\approx 8700 \mathrm{~cm}^{-1}$ and $\approx 11600 \mathrm{~cm}^{-1}$ respectively and only the first $\mathrm{O}-\mathrm{H}$ overtone at $\approx 7000 \mathrm{~cm}^{-1}$ is close to matching with the energy gap. Deuteration of $\mathrm{O}-\mathrm{H}$ did not give a significant increase as the second overtone of $\mathrm{O}-\mathrm{D}$ vibration $\left(\approx 7800 \mathrm{~cm}^{-1}\right)$ matches quite well with the energy gap, too. Seitz and coworkers $^{17}$ have already suggested that the EGL is not 
'universally relevant' as the sole consideration of the smallest energy gap in the quenching process neglects the FranckCondon principle, which governs the intensity of vibronic transitions - the exact nature of the quenching process discussed herein. This is particularly important in Ln(III) systems with multiple electronic states such as Sm(III), compared to $\mathrm{Yb}$ (III) which only has two states.

As our results suggest, the smallest energy gap is not effectively quenched by multiphonon relaxation in $\mathbf{S m - 1}$ but the energy difference between the excited ${ }^{4} \mathrm{G}_{5 / 2}$ and ${ }^{6} \mathrm{~F}_{9 / 2}$ states indeed matches well with the second $\mathrm{C}-\mathrm{H}\left(\approx 8700 \mathrm{~cm}^{-1}\right)$ and third $\mathrm{C}-\mathrm{D}$ overtones $\left(\approx 8400 \mathrm{~cm}^{-1}\right)$. Comparing the quantum yields of $\mathrm{CD}_{3} \mathrm{OD}$ and $\mathrm{CH}_{3} \mathrm{OD}$, though, still revealed a 10 -fold difference and we believe it is due to the higher probability of having three quanta of $\mathrm{C}-\mathrm{H}$ vibrational energy in resonance with $\Delta E\left({ }^{4} \mathrm{G}_{5 / 2} \rightarrow{ }^{6} \mathrm{~F}_{9 / 2}\right)$ than four C-D vibrational quanta. ${ }^{18}$

The EGL was simply not comprehensive enough to estimate the quenching processes in Sm(III) compounds due to the many possibilities of vibronic transitions amongst the various electronic states. Furthermore, the NIR transitions are of lower energies than visible light transitions therefore the overtones required to match the energy differences are less, resulting in more probable quenching, hence the generally lower quantum yields in polar solvents.

The quantum yields obtained in fluorobenzene are also quite good for both complexes. The aromaticity increases the rigidity for the complex and the absorption band deterioration problem was not observed, too, as the $\mathrm{C}-\mathrm{F}$ bond is less prone to decomposition than the $\mathrm{C}-\mathrm{Cl}$ bond. The introduction of a highly electronegative fluorine atom also rendered the solvent molecule quite polar (comparable dipole moment with methanol), yet solvent coordination to the $\operatorname{Ln}(\mathrm{III})$ center is not expected due to steric hindrance of the aromatic ring and, therefore, the quantum yields are quite good as the Ln(III) centers are well-shielded from oscillators.

An interesting finding was presented in deuterated benzene and toluene measurements of Sm-1 and Yb-1 (Table 2). The NIR luminescence quantum yields exhibited a decrease, contrary to the general notion that deuteration of oscillators often results in less quenching. However, for visible luminescence of Sm-1, the quantum yield increased as expected, intriguing us to surmise that the deuteration of aromatic $\mathrm{C}-\mathrm{H}$ leads to a preferential radiative decay of the excited energy at the ${ }^{4} G_{5 / 2}$ energy state of Sm-1 to the higher $\Delta E$ transitions, resulting in higher quantum yields than the NIR ones, although this remain a wild hypothesis.

The sensitization efficiency ( $\left.\eta_{\text {sens }}\right)$ is a parameter that could be introduced to summarize the various processes that took place between photo-absorption and luminescence. It is calculated as the ratio between the intrinsic quantum yield ( $Q_{\mathrm{Ln}}^{\mathrm{Ln}}$, luminescence quantum yield by $\mathrm{f}$-f-direct excitation) and the overall quantum yield $\left(Q_{\mathrm{L}}^{\mathrm{Ln}}\right.$, via antenna effect). While the overall quantum yield can be obtained by conventional methods such as the relative and absolutely methods, measuring the intrinsic quantum yield is impractical, though technically feasible. Fortunately, the intrinsic quantum yield could be obtained from observed and radiative lifetimes. The observed lifetime is the lifetime measured experimentally and the radiative lifetime denotes the lifetime in the absence of any non-radiative deactivation pathways. Below are the equations for calculating the sensitization efficiency and, for europium(III), the radiative lifetime, obtained by theoretical methods that are detailed in ref. 19:

$$
\begin{gathered}
\eta_{\text {sens }}=Q_{\mathrm{L}}^{\mathrm{Ln}} / Q_{\mathrm{Ln}}^{\mathrm{Ln}}=\left(Q_{\mathrm{L}}^{\mathrm{Ln}}\right)\left(\tau_{\mathrm{rad}}\right) / \tau_{\mathrm{obs}} \\
1 / \tau_{\mathrm{rad}}=A_{\mathrm{MD}, 0} n^{3}\left(I_{\mathrm{tot}} / I_{\mathrm{MD}}\right)
\end{gathered}
$$

where $A_{\mathrm{MD},{ }_{0}}$ is a constant $\left(14.65 \mathrm{~s}^{-1}\right), n$ is the refractive index and $I_{\text {tot }}$ and $I_{\mathrm{MD}}$ are the integrated intensities of the total spectrum and only the magnetic dipole transition $\left({ }^{5} \mathrm{D}_{0} \rightarrow{ }^{7} \mathrm{~F}_{1}\right)$ respectively.

The sensitization efficiencies in various solvents of Eu-1 is presented in Table 3 and assuming Sm-1 and Yb-1 are isostructural, the results can be used as reference. Surprisingly, the intrinsic quantum yields of acetonitrile and DMSO were higher than that of the non-polar solvents. The poor sensitization

\begin{tabular}{|c|c|c|c|c|c|c|}
\hline $\mathrm{CCl}_{4}$ & 0 & - & - & 43 & 31 & 75 \\
\hline Benzene & 0 & 40 & 59.5 & 53 & 32 & 61 \\
\hline $\mathrm{CHCl}_{3}$ & 1.04 & - & - & 46 & 18 & 37 \\
\hline i-PrOH & 1.56 & 29 & 59.2 & - & - & - \\
\hline $\mathrm{CH}_{2} \mathrm{Cl}_{2}$ & 1.6 & - & - & 27 & 6 & 22 \\
\hline EA & 1.78 & 13.6 & 52 & - & - & - \\
\hline Acetone & 2.88 & 23 & 31 & - & - & - \\
\hline $\mathrm{CH}_{3} \mathrm{CN}$ & 3.92 & 53.8 & 23.4 & - & - & - \\
\hline DMSO & 3.96 & 52.9 & 24.4 & - & - & - \\
\hline
\end{tabular}

Table 3 Sensitization efficiencies $\left(\eta_{\text {sens }}\right)$ of Eu-1 ${ }^{a}$

${ }^{a}$ TTA: values calculated with overall quantum yield obtained by relative method against quinine sulfate (vide supra). ILCT: Values calculated with overall quantum yield obtained by absolute method using an integrating sphere with excitation at the absorption maxima of the ILCT band >400 nm to avoid second order of excitation light overlapping with ${ }^{5} \mathrm{D}_{0} \rightarrow{ }^{7} \mathrm{~F}_{J}$ transitions. 
efficiency, however, is similar to methanol and this corroborates with the quenching by coordinated solvent molecules mentioned previously.

Moderately polar solvents have understandably decent intrinsic quantum yields and sensitization efficiencies. For benzene and toluene, the intrinsic quantum yields are decent only, but the lack of solvent-associated deactivation pathways results in higher sensitization efficiencies which compensates for the overall quantum yields. Isopropanol, on the other hand, has a moderate sensitization efficiency despite the presence of $\mathrm{O}-\mathrm{H}$ oscillators. We propose that it is a result of the relatively high viscosity of isopropanol that plays a role in hindering multiphonon quenching.

The efficiency of sensitization via the non-triplet energy transfer pathway was also investigated using an integrating sphere to measure the absolute quantum yields of Eu-1 in nonpolar solvents as there is not a common quantum yield standard excited at $c a .400 \mathrm{~nm}$, which is near the absorption maxima of the ILCT bands. Comparing between the intrinsic quantum yields of TTA and ILCT excitation in benzene and toluene, the values are $32 \%$ and $38 \%$ higher in ILCT excitation, confirming that the non-triplet sensitization pathway is more efficient in populating the excited states of $\mathrm{Eu}(\mathrm{III})$. Nevertheless, the shorter lifetime of the excited ILCT state compared to the excited triplet state of TTA would may be prone to competition from other singlet state deactivation pathways such as fluorescence.

\section{Conclusion}

In this paper, we investigated fundamental processes involved in the sensitization of visible and NIR luminescence of our $\mathrm{Eu}(\mathrm{III}), \mathrm{Sm}$ (III) and $\mathrm{Yb}$ (III) complexes with a ligand system containing 2-thenoyltrifluoroacetonates and a diethylaniline-based tridentate chromophore capable of imparting intraligand charge transfer character. This ligand system is able to sensitize all three $\operatorname{Ln}$ (III) and the photoluminescence studies of individual ions contributed to a library of findings with just one system. A detailed solvatochromic study was performed and the results were used to illustrate various processes during the antenna effect. The coordination of polar solvent molecules was evidenced in the hypersensitive transitions of Eu-1. Quantum yield measurements of $\mathbf{S m - 1}$ offered further insights on the estimating luminescence quenching regarding the energy gap law and the sensitization efficiencies of Eu-1 were determined as well. Photoluminescence and cyclic voltammetry experiments of Yb-1 provided food for thought on the energy transfer mechanism of sensitizing $\mathrm{Yb}(\mathrm{III})$ luminescence via a non-triplet pathway by the ILCT band.

\section{Experimental}

\section{Materials and methods}

2-Thenoyltrifluoroacetonate (tta) lanthanide salt Eu(tta) $)_{3} \cdot 2 \mathrm{H}_{2} \mathrm{O}$ was purchased from Alfa Aesar. $\mathrm{Sm}(\mathrm{tta})_{3} \cdot 2 \mathrm{H}_{2} \mathrm{O}$ and $\mathrm{Yb}(\mathrm{tta})_{3}{ }^{-}$ $\cdot 2 \mathrm{H}_{2} \mathrm{O}$ were synthesized according to literature procedures with anhydrous $\mathrm{SmCl}_{3}$ (99.99\%), anhydrous $\mathrm{YbCl}_{3}(99.99 \%)$ and 2thenoyltrifluoroacetone (99\%) from Aldrich. THF was dried with sodium metal and benzophenone. Spectrophotometric grade solvents, sulfuric acid concentrate and quinine sulfate were purchased from Sigma-Aldrich and deuterated solvents were purchased from Cambridge Isotope Laboratories and SigmaAldrich.

\section{Synthetic procedures}

The synthesis of Sm-1 was reported in ref. 10 and is adopted for the syntheses of Eu-1 and Yb-1.

Synthesis of $1.0 .134 \mathrm{~g}$ of magnesium turnings $(5.5 \mathrm{mmol})$ and catalytic amount of iodine was added into $5 \mathrm{~mL}$ of dry THF. $1.14 \mathrm{~g}$ of 4-bromo- $N, N$-dimethylaniline ( $5 \mathrm{mmol}$ ) dissolved in 15 $\mathrm{mL}$ of dry THF was added slowly at room temperature and further refluxed for 3 hours to obtain the Grignard reagent. The cooled Grignard reagent was then added slowly into a $30 \mathrm{~mL}$ dry THF solution with $1 \mathrm{~g}$ of cyanuric chloride $(5.4 \mathrm{mmol})$ dissolved at $4{ }^{\circ} \mathrm{C}$. The reaction mixture was stirred for an additional 4 hours at room temperature before being quenched with saturated ammonium chloride solution. The precipitate was filtered and the solution was evaporated. The crude was redissolved in diethyl ether and extracted against water $(3 \times)$, brine, dried over anhydrous $\mathrm{MgSO}_{4}$ and concentrated in vacuo. The product was obtained after purification by flash column chromatography with $\mathrm{PE}: \mathrm{DCM} / 5: 1$. The product was further recrystallized in $\mathrm{PE}$ if it contains a blue-fluorescent purity under a handheld UV lamp excited at $254 \mathrm{~nm}$. Yield: $57 \%$. $\delta_{\mathrm{H}}\left(400 \mathrm{MHz} ; \mathrm{CDCl}_{3}\right): 8.32$ (m, 2H, ArH), $6.82(\mathrm{~m}, 2 \mathrm{H}, \mathrm{ArH}), 3.49\left(\mathrm{q}, J=7.2 \mathrm{~Hz}, 4 \mathrm{H}, \mathrm{CH}_{2}\right)$, $1.25 \mathrm{ppm}\left(\mathrm{t}, J=7.2 \mathrm{~Hz}, 6 \mathrm{H}, \mathrm{CH}_{3}\right) . \delta_{\mathrm{C}}: 173.74,170.87,152.74$, $132.54,118.60,111.02,44.80,12.57$ ppm. MS (ESI $\left.{ }^{+}\right): 296[(\mathrm{M}-$ $\left.\mathrm{H})^{+}\right], 319\left[(\mathrm{M}+\mathrm{Na})^{+}\right]$.

Synthesis of $2.0 .102 \mathrm{~g}$ of pyrazole $(1.5 \mathrm{mmol})$ was dissolved into $10 \mathrm{~mL}$ of dry THF and $108 \mathrm{mg}$ of potassium metal (1.4 mmol) was added. The reaction mixture was refluxed until the potassium disappeared. The deprotonated species was transferred under nitrogen a $10 \mathrm{~mL}$ of dry THF solution containing $0.3 \mathrm{~g}$ of 1 at room temperature. The resulting yellow reaction mixture was refluxed overnight, filtered and evaporated. The crude was redissolved in EA and extracted against water, brine, dried over anhydrous $\mathrm{MgSO}_{4}$ and concentrated in vacuo. The yellow crude was purified with column chromatography with DCM : EA/100 : 1 to elute a yellow product. Yield: $48 \% . \delta_{\mathrm{H}}(400$ MHz; $\left.\mathrm{CDCl}_{3}\right): 8.79(\mathrm{~m}, 2 \mathrm{H}, \mathrm{ArH}), 8.53(\mathrm{~d}, 2 \mathrm{H}, \mathrm{py}), 7.92(\mathrm{~s}, 2 \mathrm{H}, \mathrm{py})$, $6.71(\mathrm{~d}, 2 \mathrm{H}, \mathrm{py}), 6.54(\mathrm{~m}, 2 \mathrm{H}, \mathrm{ArH}), 3.48\left(\mathrm{q}, J=7.2 \mathrm{~Hz}, 4 \mathrm{H}, \mathrm{CH}_{2}\right)$, $1.24 \mathrm{ppm}\left(\mathrm{t}, J=7.2 \mathrm{~Hz}, 6 \mathrm{H}, \mathrm{CH}_{3}\right) . \delta_{\mathrm{C}}: 174.26,162.67,152.09$, 144.92, 131.99, 130.16, 120.36, 110.66, 109.3, 44.65, 12.59 ppm. MS $\left(\operatorname{ESI}^{+}\right): m / z 383\left[(\mathrm{M}+\mathrm{Na})^{+}\right], 743\left[(2 \mathrm{M}+\mathrm{Na})^{+}\right]$.

Syntheses of $\mathrm{Ln}(\mathrm{tta})_{3} \cdot \mathbf{2 H}_{2} \mathrm{O}^{20} 2 \mathrm{~g}$ of thenoyltrifluoroacetone $(9 \mathrm{mmol}$ ) was dissolved in $30 \mathrm{~mL}$ of absolute ethanol and $9 \mathrm{~mL}$ of $1 \mathrm{M}$ of ammonium hydroxide was added. The mixture was allowed to stir until clear and $3 \mathrm{mmol}$ of lanthanide(III) chloride in $36 \mathrm{~mL}$ of water was added. Stirring was stopped upon complete addition and the mixture was allowed to stand and solidify for several hours. The solution was removed and the solid was washed with water and subsequently dried in a vacuum desiccator overnight. The solids were dissolved in 4.5 $\mathrm{mL}$ of acetone and precipitated with $36 \mathrm{~mL}$ of deionized water. 
The solution was removed and the thick oil was allowed to solidify. The solid was then dried under high vacuum at room temperature and recrystallized in petroleum ether to remove excess TTA. Yield: $24 \%$. HRMS $\left(\mathrm{ESI}^{+}\right): m / z 859.8960\left\{\left[\mathrm{Yb}(\mathrm{tta})_{3}+\right.\right.$ $\left.\mathrm{H}]^{+}\right\}, 815.8710\left\{\left[\mathrm{Sm}(\mathrm{tta})_{3}+\mathrm{H}\right]^{+}\right\}$.

Syntheses of $\mathbf{L n}-1 . \operatorname{Ln}(\mathrm{tta})_{3} \cdot 2 \mathrm{H}_{2} \mathrm{O}$ and the ligand was mixed in a $1: 1$ ratio in a solution of methanol and stirred at $50{ }^{\circ} \mathrm{C}$ overnight. The solvent was subsequently evaporated and the residue was re-dissolved in minimum amount of diethyl ether and precipitated by $n$-hexanes. The bright yellow complex was obtained by repeated precipitation thrice. Average yield: $80 \%$.

Eu-1. HRMS (ESI) $)^{+}: m / z \quad 955.0798\left\{\left[\mathrm{Eu}(\mathrm{tta})_{2}\right]^{+}\right\}$. Elemental analysis (\%) calcd for $\mathrm{C}_{47} \mathrm{H}_{43} \mathrm{EuF}_{9} \mathrm{~N}_{8} \mathrm{O}_{6} \mathrm{~S}_{3}$ : C, 45.71; H, 3.51; N, 9.07. Found: C, 45.15; H, 3.08; N, 8.80.

Sm-1. MS (ESI) ${ }^{+}: m / z 954\left\{\left[\mathrm{Sm}(\mathrm{tta})_{2}\right]^{+}\right\}$. Elemental analysis (\%) calcd for $\mathrm{C}_{47} \mathrm{H}_{43} \mathrm{SmF}_{9} \mathrm{~N}_{8} \mathrm{O}_{6} \mathrm{~S}_{3}$ : C, 45.77; H, 3.51; N, 9.08. Found: C, 45.89; H, 3.37; N, 8.91.

Yb-1. MS (ESI) ${ }^{+}: m / z 976\left\{\left[\mathrm{Yb}(\mathrm{tta})_{2}\right]^{+}\right\}$. Elemental analysis (\%) calcd for $\mathrm{C}_{47} \mathrm{H}_{43} \mathrm{YbF}_{9} \mathrm{~N}_{8} \mathrm{O}_{6} \mathrm{~S}_{3}: \mathrm{C}, 44.94 ; \mathrm{H}, 3.45 ; \mathrm{N}, 8.92$. Found: C, 43.52; H, 3.12; N, 8.80.

\section{Photophysical measurements}

Solution samples of $c a$. 0.1 and 0.3 absorbances at $350 \mathrm{~nm}$ were prepared for visible and NIR photoluminescence measurements respectively. Measurements were prepared in the unit of absorbance instead of concentration as the relative absorbances at $350 \mathrm{~nm}$ are slightly different for the complexes in different solvents due to the ILCT character. The concentrations at 0.1 and 0.3 absorbances are estimated to be $c a .1 .4$ to $1.8 \mu \mathrm{M}$ and 4.3 to $5.4 \mu \mathrm{M}$ respectively. The concentrations are low enough to avoid inner filter effect. To avoid time-dependent solvent coordination and instability issues (Fig. S76-S79†), each sample solution was freshly prepared from solid sample. Separate samples were used for (1) UV-vis, emission and excitation scans; (2) luminescence lifetime measurements and (3) quantum yield measurements.

All room temperature solution measurements were done in quartz cuvettes of $1 \mathrm{~cm}$ path length. UV-vis spectra were recorded with an HP UV-8453 spectrophotometer. Room temperature photoluminescence measurements data obtained with (1) Edinburgh Instruments FLSP920 spectrophotometer equipped with a Xe900 continuous xenon lamp $(450 \mathrm{~W})$, xenon flashlamp $\left(60 \mathrm{~W}\right.$ ) and a Hamamatsu R928P cooled at $-20{ }^{\circ} \mathrm{C}$ (for visible emission) and (2) PTI QuantaMaster ${ }^{\mathrm{TM}} 50$ equipped with a $75 \mathrm{~W}$ xenon arc lamp, double emission monochromator using 400 $\mathrm{nm}$ blazed 1200 lines per mm or $1200 \mathrm{~nm}$ blazed 600 lines per mm gratings, a Hamamatsu R928 PMT (for visible emission) and a Hamamatsu R5108 PMT (for visible and/or NIR emission), both thermoelectrically cooled. Low temperature (77 K) measurements were measured on FLSP920 using an EPR dewar from Edinburgh Instruments. Samples were dissolved in 2methyltetrahydrofuran, inserted into an EPR quartz sample rod and cooled with liquid nitrogen. Emission spectra were recorded at 30 min intervals until the intensity and emission profiles remained constant ( $\approx 2$ hours) and the spectra were taken as final. Visible emission spectra obtained were corrected for the spectral responses and NIR emission spectra were not corrected due to the weak intensities and high noise levels.

\section{Luminescence lifetimes and quantum yields}

Luminescence lifetimes of visible emissions were measured with FLSP290 and fitted with Origin. Luminescence quantum yields were measured relative to quinine sulfate in $0.1 \mathrm{M}$ sulfuric acid $\left(\lambda_{\mathrm{ex}}=350 \mathrm{~nm}, \Phi=0.577\right)^{21}$ for visible emission and to $\left[\mathrm{Yb}(\mathrm{tta})_{3}\left(\mathrm{H}_{2} \mathrm{O}\right)_{2}\right]$ in toluene $\left(\lambda_{\text {ex }}=340 \mathrm{~nm}, \Phi=0.35 \%\right)^{22}$ for NIR emissions. These standards were chosen as the excitation and emission wavelengths match well with our samples. Absolute quantum yields were measured using an integrating sphere from Edinburgh Instruments and the quantum yield values were obtained using the F900 software. All photophysical measurements were averages of triplicates.

\section{Cyclic voltammetry}

Electrochemical study of the $\mathbf{S m - 1}$ and $\mathbf{Y b - 1}$ complexes was performed on a CHI $1030 \mathrm{~A}$ instrument and the electrochemical cell was of conventional design. A glassy carbon disk $(3 \mathrm{~mm}$ diameter) was used as working electrode, a Pt wire as counter electrode and a non-aqueous $\mathrm{Ag} / \mathrm{AgNO}_{3}$ as reference electrode. All the electrochemical experiments were performed using 0.1 $\mathrm{M}$ tetrabutylammonium hexafluorophosphate (TBHP) in tetrahydrofuran and the solution was purged with argon. Ferrocene (Fc) was used as the internal standard, and all potentials are referenced to the ferrocene/ferrocenium $\left(\mathrm{Fc} / \mathrm{Fc}^{+}\right)$couple. All scans were done at $100 \mathrm{mV} \mathrm{s}^{-1}$.

\section{Acknowledgements}

We thank Dr L. Y. S. Lee and Dr C.-S. Tsang of our department for performing the cyclic voltammetry experiments, and for their useful discussion. Financial support from the Hong Kong Research Grants Council (PolyU 153012/15P), Hong Kong Polytechnic University (G-UC08 and 1-ZVGG), and the University Central Facility for Chemical and Environmental Analysis (UCEA) are gratefully acknowledged. WS Lo acknowledges the receipt of a postgraduate studentship from the Hong Kong Polytechnic University.

\section{References}

1 (a) J.-C. G. Bünzli and C. Piguet, Chem. Soc. Rev., 2005, 34, 1048-1077; (b) E. G. Moore, A. P. S. Samuel and K. N. Raymond, Acc. Chem. Res., 2006, 39, 53-61; (c) S. V. Eliseeva and J.-C. G. Bünzli, Chem. Soc. Rev., 2010, 39, 189-227; (d) J.-C. G. Bünzli and S. V. Eliseeva, Chem. Sci., 2013, 4, 1939-1949; (e) L. Sun, X. Ge, J. Liu, Y. Qiu, Z. Wei, B. Tian and L. Shi, Nanoscale, 2014, 6, 13242-13252.

2 T. Förster, Discuss. Faraday Soc., 1959, 27, 7-17.

3 D. L. Dexter, J. Chem. Phys., 1953, 21, 836-850.

4 A. Beeby, I. M. Clarkson, R. S. Dickins, S. Faulkner, D. Parker, L. Royle, A. S. de Sousa, J. A. Gareth Williams and M. Woods, J. Chem. Soc., Perkin Trans. 2, 1999, 493-504. 
5 J.-C. G. Bünzli and S. V. Eliseeva, in Lanthanide Luminescence - Photophysical, Analytical and Biological Aspects, ed. P. Hänninen and H. Härmä, Spring, Verlag, Berlin, Heidelberg, 2011, vol. 7, pp. 1-46.

6 (a) F. R. Gonçalves e Silva, O. L. Malta, C. Reinhard, H.-U. Güdel, C. Piguet, J. E. Moser and J.-C. G. Bünzli, J. Phys. Chem. A, 2002, 106, 1670-1677; (b) K. Lunstroot, P. Nockermann, K. Van Hecke, L. Van Meervelt, C. GörllerWalrand, K. Binnemans and K. Driesen, Inorg. Chem., 2009, 48, 3018-3026; (c) A. T. Bui, A. Grichine, S. Brasselet, A. Duperray, C. Andraud and O. Maury, Chem.-Eur. J., 2015, 21, 17757-17761; (d) Y. Liu, L. Sun, J. Liu, Y.-X. Peng, X. Ge, L. Shi and W. Huang, Dalton Trans., 2015, 44, 237-246. 7 (a) W. D. Horrocks Jr, J. P. Bolender, W. D. Smith and R. M. Supkowski, J. Am. Chem. Soc., 1997, 119, 5972-5973; (b) S. Faulkner, B. P. Burton-Pye, T. Khan, L. R. Martin, S. D. Wray and P. J. Skabara, Chem. Commun., 2002, 16681669; (c) R. F. Ziessel, G. Ulrich, L. Charbonnière, D. Imbert, R. Scopelliti and J.-C. G. Bünzli, Chem.-Eur. J., 2006, 12, 5060-5067; (d) N. M. Shavaleev, R. Scopelliti, F. Gumy and J.-C. G. Bünzli, Inorg. Chem., 2009, 48, 29082918; (e) T. Lazarides, M. A. H. Alamiry, H. Adams, S. J. A. Pope, S. Faulkner, J. A. Weinstein and M. D. Ward, Dalton Trans., 2007, 1484-1491.

8 K. Binnemans, Chem. Rev., 2009, 109, 4283-4374.

9 (a) C. Yang, L. Fu, Y. Wang, J. P. Zhang, W.-T. Wong, X.-C. Ai, Y.-F. Qiao, B.-S. Zou and L.-L. Gui, Angew. Chem., Int. Ed., 2004, 43, 5010-5013; (b) L.-M. Fu, X.-C. Ai, M.-Y. Li, X.-F. Wen, R. Hao, Y.-S. Wu, Y. Wang and J.-P. Zhang, J. Phys. Chem. A, 2010, 114, 4494-4500.
10 W.-S. Lo, J. Zhang, W.-T. Wong and G.-L. Law, Inorg. Chem., 2015, 54, 3725-3727.

11 C. Reichardt and T. Welton, in Solvents and Solvent Effects in Organic Chemistry, Wiley-VCH Verlag GmbH \& Co. KGaA, Weinheim, Germany, 4th edn, 2011.

12 D. Parker, Coord. Chem. Rev., 2000, 205, 109-130.

13 M. Latva, H. Takalo, V.-M. Mukkala, C. Matachescu, J. C. Rodriguez-Ubis and J. Kankare, J. Lumin., 1997, 75, 149-169.

14 D. Rehm and A. Weller, Isr. J. Chem., 1970, 8, 259-271.

15 P. Kadjane, L. Charbonnière, F. Camerel, P. P. Lainé and R. Ziessel, J. Fluoresc., 2008, 18, 119-129.

16 (a) R. Englman and J. Jortner, Mol. Phys., 1970, 18, 145-164; (b) G. W. Robinson and R. P. Frosch, J. Chem. Phys., 1963, 38, 1187-1203.

17 C. Doffek, J. Wahsner, E. Kreidt and M. Seitz, Inorg. Chem., 2014, 53, 3263-3265.

18 C. Doffek, N. Alzakhem, C. Bischof, J. Wahsner, T. GüdenSilber, J. Lügger, C. Platas-Iglesias and M. Seitz, J. Am. Chem. Soc., 2012, 134, 16413-16423.

19 A. Aebischer, F. Gumy and J.-C. G. Bünzli, Phys. Chem. Chem. Phys., 2009, 11, 1346-1353.

20 R. G. Charles and R. C. Ohlmann, J. Inorg. Nucl. Chem., 1965, 27, 255-259.

21 J. W. Eastman, Photochem. Photobiol., 1967, 6, 55-72.

22 S. B. Meshkova, Z. M. Topilova, D. V. Bolshoy, S. V. Beltyukova, M. P. Tsvirko and V. Y. Venchikov, Acta Phys. Pol., A, 1999, 95, 983-990. 